Polymer Journal Vol. 1, No. 3, pp. 304-311 (1970)

\title{
Comparison of Various Methods of Determining Molecular Weight Distribution
}

\author{
Atsushi Yamamoto, Ichiro Noda, and Mitsuru Nagasawa \\ Department of Synthetic Chemistry, Faculty of Engineering, \\ Nagoya University, Chikusa-ku, Nagoya, Japan.
}

(Received December 5, 1969)

\begin{abstract}
Resolutions of various methods used to determine molecular weight distribution of linear polymers, such as the precipitation chromatography, elution sedimentation velocity, and gel permeation chromatography methods, were compared by determining molecular weight distributions of samples obtained by mixing two samples of monodisperse poly $(\alpha$-methylstyrene). The sedimentation velocity method showed the double peaks in the molecular weight distributions slightly better than the column fractionation method, and but there was no appreciable difference between the resolution of the elution method and that of precipitation chromatography. However, gel permeation chromatography appears to have much lower resolution than the other methods if no correction for broadening effect is made.

KEY WORDS Molecular Weight Distribution / Precipitation Chromatography / Elution / Column Fractionation / Sedimentation / Gel Permeation Chromatography / Poly ( $\alpha$-Methylstyrene) /
\end{abstract}

Gel permeation chromatography developed by Moore $^{1}$ is now widely used as a convenient method of determining the molecular weight distribution of linear polymers. The method is quick and has good reproducibility. However, it is also well known that its resolution is rather limited, particularly if the polymer has a narrow molecular weight distribution. Even though a sample is ideally monodisperse, its chromatogram appears not as a straight line but as a bell-shaped Gaussian curve. Although methods to correct this broadening effect have been proposed, ${ }^{2-4}$ the application of those methods cannot be standardized because of the uncertainty in assessing the resolution factor i.e., the width of the Gaussian curve of an ideally monodisperse polymer. In view of the resolution factor limitation in respect to GPC, therefore, the effectiveness of various other methods so far used such as the column fractionation and sedimentation velocity methods are worth closer attention when the sample has a narrow molecular weight distribution, or has a discontinuity in its molecular weight distribution.
The purpose of this paper is to compare the resolutions of the precipitation chromatography, elution, sedimentation velocity and gel permeation chromatography methods for narrow molecular weight distributions. Emphasis is given to the comparison between the precipitation chromatography and elution methods, that is, to the effect of the temperature gradient created along the fractionation column on resolution of the column. The former is generally regarded to be superior to the latter but no definite conclusion has yet been obtained.

Comparison is made by determining molecular weight distributions of mixtures of two monodisperse $\operatorname{poly}(\alpha$-methylstyrene) samples each having a very narrow molecular weight distribution. ${ }^{5-7}$ The samples have such narrow molecular weight distributions that the mixtures must have binodial molecular weight distributions even when the ratio of weight average molecular weight to number average molecular weight of the mixture is as low as 1.02 . Therefore, if a method of molecular weight distribution determination has a high resolution, the molecular weight distribution obtained must 
show double peaks.

\section{EXPERIMENTAL}

\section{Samples}

The samples were prepared by anionic polymerization of $\alpha$-methylstyrene with $n$-butyl lithium in $\mathrm{THF}^{5}$ The molecular weights of the samples are listed in Table I. If we calculate the ratios of weight average molecular weight to number average molecular weight of those monodisperse samples from their molecular weight distributions, they are certainly smaller than 1.01. ${ }^{5}$ Samples Nos. 9-12 were prepared by mixing almost equal amounts of two monodisperse samples. Mixture No. 11 as well as Nos. 9 and 10 show clear double peaks in their sedimentation patterns, ${ }^{6}$ though the molecular weights of the components of mixture No. 12 are too close to be distinguished even in the sedimentation pattern.

Table I. Samples

A. Monodisperse $\operatorname{poly}(\alpha$-methylstyrene)

\begin{tabular}{cccc}
\hline No. & $M_{n} \times 10^{-4}$ & $M_{w} \times 10^{-4}$ & $M_{v} \times 10^{-4}$ \\
\hline 1 & 5.7 & & 5.7 \\
2 & 14.2 & & 12.8 \\
3 & & & 24.0 \\
4 & $34.2^{\mathrm{a}}$ & $34.2^{\mathrm{a}}$ & 33.7 \\
5 & $43.3^{\mathrm{a}}$ & $43.3^{\mathrm{a}}$ & 43.0 \\
6 & & & 46.0 \\
7 & $50.0^{\mathrm{a}}$ & & \\
8 & $68.0^{\mathrm{a}}$ & $69.4^{\mathrm{a}}$ & \\
\hline
\end{tabular}

\begin{tabular}{rlc}
\hline B. Mixtures & & \\
\hline No. & $\begin{array}{c}\text { Constituents } \\
(50: 50)\end{array}$ & $M_{w} / M_{n}$ b \\
\hline 9 & No. $2+$ No. 4 & 1.27 \\
10 & No. $3+$ No. 6 & 1.11 \\
11 & No. $4+$ No. 7 & 1.04 \\
12 & No. $4+$ No. 5 & 1.02 \\
\hline
\end{tabular}

a These molecular weights were determined after the high and low molecular weight tails were removed by fractionation. Therefore the molecular weights of the samples used may differ slightly from these values.

b These values were calculated from the molecular weights of the constituents, assuming that the constituents were ideally monodisperse.

\section{Molecular Weight Determination}

The number average molecular weight $M_{n}$ was determined in toluene at $25^{\circ} \mathrm{C}$ with a Hewllet-Packard high-speed membrane osmometer type 502. The weight average molecular weight $M_{w}$ was determined in cyclohexane and toluene with a modified light-scattering instrument from Shimadzu Seisakusho. The viscosity average molecular weights $M_{v}$ of monodisperse samples and their blends were calculated from a reduced viscosity in toluene at a single concentration of about $0.2 \mathrm{~g} / \mathrm{d} l$ using the following equations. $^{5}$

$$
\eta_{s p} / C=[\eta]+0.356[\eta]^{2} C
$$

and

$$
[\eta]=1.01 \times 10^{4} M_{w}{ }^{0.72}
$$

where $C$ is given by $\mathrm{g} / \mathrm{d} l$ and the temperature is $25^{\circ} \mathrm{C}$. When the molecular weight was as high as $5 \times 10^{5}$, the molecular weight value was read off on the experimental plot between $\log [\eta]$ and $\log M_{w} \cdot{ }^{5} \quad$ Viscosity was measured in a Ubbelohde-type viscometer designed for small quantities of the sample.

\section{Precipitation Chromatography}

The method used was one developed by Baker and Williams ${ }^{9}$ and modified by Schneider, Holmes, Mijal and Loconti, ${ }^{10}$ which combines the use of a thermal gradient with a solvent gradient to achieve a chromatographic process. The essential part of the apparatus is similar to Figure 1 of ref. 10. The effective length and inner diameter of the glass column are $140 \mathrm{~cm}$ and $3.67 \mathrm{~cm}$, respectively. The thermal gradient was introduced by heating the top and cooling the bottom of a metal (aluminum) tube which enclosed the glass column for fractionation. The heating and cooling were done with circulating water regulated at $58 \pm 0.1^{\circ} \mathrm{C}$ and $10+0.1^{\circ} \mathrm{C}$, respectively. Thus, the temperature gradient set up in the column, which was measured by thermocouples inserted into the aluminum wall, was $0.48^{\circ} \mathrm{C} / \mathrm{cm}$. Thermal contact between the metal tube and the glass column was achieved with the use of a silicone oil. According to a study made by Schulz, ${ }^{11}$ the temperature difference between the glass wall and the center of the column may be about $1{ }^{\circ} \mathrm{C}$. The polymercoated glass beads occupy the uniform high temperature region at the top 


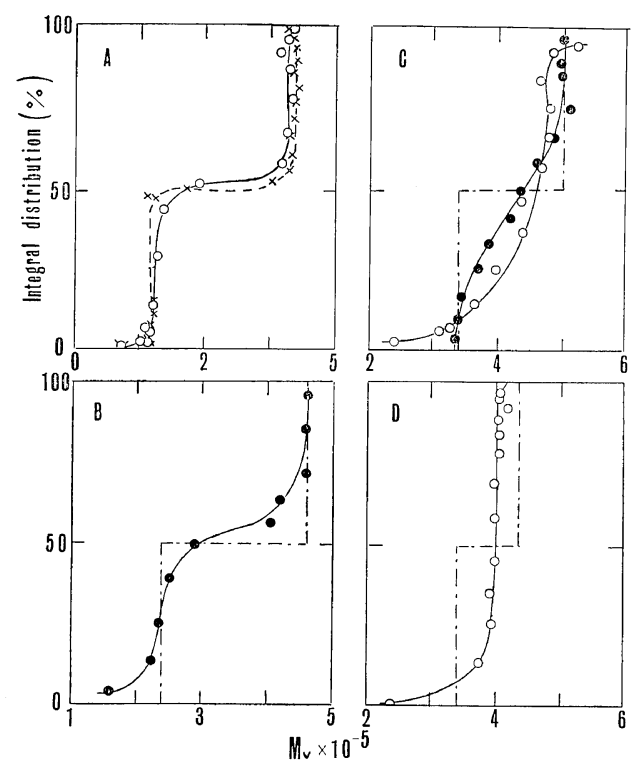

Figure 1. Comparison of the integral molecular weight distributions determined by precipitation chromatography and the elution method.

Open and filled circles denote the fractionation results for the mixture by the precipitation chromatography and elution method, respectively. Crosses denote the fractionation results of the constituent components by precipitation chromatography. Chain lines denote the molecular weight distribution of the mixture calculated from those of the constituent components, assuming ideal monodispersity of the components.

of column.

The size of the glass beads is between 170 mesh $(0.088 \mathrm{~mm}$ diameter $)$ and 115 mesh $(0.125$ $\mathrm{mm}$ diameter). They were purified with $\mathrm{HCl}$, concentrated $\mathrm{HNO}_{3}$ and acetone. About 0.5 to $1.0 \mathrm{~g}$ of the sample was dissolved in chloroform, and then methanol was added to the solution until it was on the point of becoming turbid. The total volume of chloroform and methanol was adjusted to $50 \mathrm{~m} l$ which was almost equal to the hold-up volume of the glass beads (about $130 \mathrm{cc}$ ), and the glass beads were added to the solution. The solution was slowly evaporated for one week at room temperature, and the glass beads coated by the polymer sample were dried out in vacuo for three hours. They were placed in the column as a slurry with $n$-hexane, after being passed through a sieve of 100 mesh
(0.149 $\mathrm{mm}$ diameter). Examination under a microscope revealed that the beads were not uniformly coated with the polymer, as reported by Schneider et al. ${ }^{12}$

The elution of the polymer was made with benzene as solvent and $n$-hexane as non-solvent. Initially the column was filled with degassed $n$ hexane. The benzene used was treated with concentrated $\mathrm{H}_{2} \mathrm{SO}_{4}$ and dried with $\mathrm{CaCl}_{2}$, followed by distillation. The $n$-hexane was also distilled after drying with $\mathrm{CaCl}_{2}$, but its purity was checked by gas chromatography. The mixing of solvent and non-solvent was carried out in a mixing vessel of $1230 \mathrm{ml}$ and, hence, the solvent gradient was logarithmic in terms of time, as confirmed by the refractive index. The flow rate of solvent was regulated with a constant flow rate pump to $25 \pm 1 \mathrm{ml} / \mathrm{hr}$. A mixture of 81 vol $\%$ and 19 vol $\% n$-hexane was used as the solvent.

The elute from the column was collected every $17 \mathrm{~m} l$ in fraction-collector tubes, some of which were recombined into about 15 fractions. Each fraction was transfered into an aluminum cup, dried carefully in vacuo and then redissolved in benzene for freeze-drying. After freeze-drying, the sample was dried out in vacuo to a constant weight. Recovery of polymer exceeded $98 \%$.

\section{Elution Method}

The apparatus for the elution method was similar to that used in precipitation chromatography, differing only in that the temperature over the whole column was kept at a constant temperature between $22^{\circ} \mathrm{C}$ and $10^{\circ} \mathrm{C}$ with circulating water. All glass beads in the column were coated with sample polymer by the procedure described above and the sample was fractionated in cyclohexane by changing the temperature of cyclohexane from $10^{\circ} \mathrm{C}$ to $22^{\circ} \mathrm{C}$ stepwisely.

The fractionation data from both the precipitation chromatography and elution methods were converted to molecular weight distributions by the method of Schulz. ${ }^{13}$

\section{Gel Permeation Chromatography}

A Waters Assoc. Model 200 was employed for gel permeation chromatography. Runs were made with the combination of columns $7 \times 10^{6}$, $1 \times 10^{6}, 1 \times 10^{5}$ and $1 \times 10^{4} \mathrm{~A}$. The experi- 
Table II. Fractionation of monodisperse No. 4 by precipitation chromatographya

\begin{tabular}{cccc}
\hline $\begin{array}{c}\text { Fraction } \\
\text { No. }\end{array}$ & $\begin{array}{c}\text { Weight of } \\
\text { fraction }(\mathrm{mg})\end{array}$ & {$[\eta](\mathrm{d} l / \mathrm{g})$} & $M_{v} \times 10^{-5}$ \\
\hline 1 & 7.68 & $0.56_{3}$ & $1.7_{3}$ \\
2 & 33.15 & $0.82_{7}$ & $3.0_{4}$ \\
3 & 40.01 & $0.87_{7}$ & $3.2_{8}$ \\
4 & 47.68 & $0.88_{1}$ & $3.3_{4}$ \\
5 & 48.58 & $0.88_{1}$ & $3.3_{4}$ \\
6 & 45.51 & $0.88_{5}$ & $3.3_{5}$ \\
7 & 43.28 & $0.89_{0}$ & $3.3_{7}$ \\
8 & 40.82 & $0.89_{8}$ & $3.4_{3}$ \\
9 & 36.46 & $0.88_{2}$ & $3.3_{4}$ \\
10 & 34.66 & $0.89_{5}$ & $3.4_{1}$ \\
11 & 31.28 & $0.89_{2}$ & $3.3_{8}$ \\
12 & 17.29 & $0.88_{5}$ & $3.3_{5}$ \\
13 & 17.29 & $0.89_{9}$ & $3.2_{6}$ \\
14 & 1.99 & & \\
& Total 452.65 & &
\end{tabular}

a Sample amount, $0.4598 \mathrm{~g}$; Yield, 98.9\%; Temperature gradient, $57.1-10.6^{\circ} \mathrm{C}$; Flow rate, $26 \mathrm{ml} /$ hr; $C_{\max }, 0.53$ vol $\%$.

mental conditions used were; flow time $1 \mathrm{ml} /$ min, polymer concn. $0.5 \mathrm{~g} / \mathrm{d} l$, solvent $\mathrm{THF}$, injection time $60 \mathrm{sec}$, temp. $35 \pm 1^{\circ} \mathrm{C}$. The calibration curve for poly ( $\alpha$-methylstyrene) in THF was constructed with samples No. 1, 2, 4 and 8 . In this work we did not determine the optimum experimental conditions for the poly( $\alpha$-methylstyrene)-THF system, but simply used the conditions appropriate for poly styrene-THF systems, as advised by $\mathrm{Mr}$. Okamoto, in the laboratory of Professor A. Kotera of the Tokyo University of Education. However, a good linear relationship was found between $\log M_{v}$ and peak retention volume and, moreover, the binodial peaks of the blended samples are well separated, as can be seen later. Therefore these experimental conditions also appear to be appropriate for the present system.

\section{Sedimentation Velocity Method}

All sedimentation experiments were carried out in a $\theta$-solvent, i.e., in cyclohexane at $35 \pm$ $0.2^{\circ} \mathrm{C},{ }^{14}$ using a Spinco model $\mathrm{E}$ ultracentrifuge with a schlieren optical system and a phase plate. Runs were made at $42040 \mathrm{rpm}$ for monodisperse samples and at $59780 \mathrm{rpm}$ for mixtures, using double sector cells of $12 \mathrm{~mm}$ thickness. The molecular weight distribution was calculated from sedimentation boundary patterns taken at a single concentration between
0.1 and $0.25 \mathrm{~g} / \mathrm{d} l$ using a method slightly modified from that of Kotaka and Donkai. ${ }^{15}$ However, no correction for pressure effect was made since such a correction is irrelevant so far as the purpose of the present work is concerned. Moreover, there is as yet no satisfactory method to take into account the pressure effect if the sample is polydisperse. Measurements of the distance on the sedimentation boundary photographs were carried out on the viewing screen of a universal contour projector from Toshiba Denki Seisakusho after enlarging the pattern by a factor of 20 .

\section{RESULTS AND DISCUSSION}

\section{Column Fractionations}

As explained in the introduction, equal amounts of two poly( $\alpha$-methylstyrene) samples, both having very narrow molecular weight distributions, were mixed and fractionated by column to determine the molecular weight distribution of the mixture. The molecular weight distribution obtained was compared with that built up from the molecular weight distributions of the components. In Figure 1-A, comparison is made for sample No. 9 in which the molecular weights of both components are sufficiently far apart $\left(M_{w} / M_{n}=1.27\right)$. The molecular weight distribution of the mixture determined agrees with the molecular weight distribution calculated from those of the components which are also determined by precipitation chromatography. In Figure 1-B, the molecular weight distribution of a mixture with $M_{w} / M_{n}=1.11$ (No. 10) is also clearly shown by the elution method. However, if $M_{w} / M_{n}$ is as low as 1.04 and 1.02, their molecular weight distributions can be shown neither by elution chromatography nor by precipitation chromatography, as shown in Figures 1-C and D. From these comparisons, we may conclude that: (a) If a sample has a molecular weight distribution narrower than $M_{w} / M_{n}=1.1$ in this range of molecular weight, column fractionation would not be effective. (b) No appreciable difference can be observed between the resolution of precipitation chromatography and that of the elution method under the present experimental conditions.

It is generally believed that precipitation 


\section{A. Yамамото, I. Noda, and M. Nagasawa}

chromatography provides superior resolution, usually without questioning the effectiveness of the thermal gradient. ${ }^{16}$ In practice, the superiority of precipitation chromatography over the elution method was on occasion confirmed experimentally, but the difference between the two methods was less than expected from an elementary consideration of the operation of the columns. ${ }^{12}$ To clarify the reason behind the unexpectedly small difference between the two methods, an analysis was made of molecular weight and the amount of polymer deposited on the glass beads during the process of precipitation chromatography. In precipitation chromatography, it is assumed that the polymers dissolved at a higher temperature region of the column are deposited on the glass beads at a lower temperature. Therefore, if the solvent supply is stopped when the solvent is increased by an amount sufficient to develop the sample through the whole column, and the solution is pushed out of the column with nitrogen gas at the same speed, the polymer molecules could

Table III. Molecular weight and amount of sample deposited on glass beads during the process of precipitation chromatographya

\begin{tabular}{cccc}
\hline $\begin{array}{c}\text { Section } \\
\text { No. }\end{array}$ & $\begin{array}{c}\text { Weight } \% \text { of } \\
\text { polymer deposited }\end{array}$ & {$[\eta](\mathrm{d} l / \mathrm{g})$} & $M_{v} \times 10^{-4}$ \\
\hline 1 & 32.5 & 0.87 & 32.6 \\
2 & 12.0 & 0.88 & 32.8 \\
3 & 6.4 & 0.85 & 31.6 \\
4 & 1.9 & 0.63 & 20.2 \\
5 & 1.1 & - & - \\
6 & 4.8 & 0.43 & 11.7 \\
7 & 12.5 & 0.46 & 12.7 \\
8 & 9.8 & 0.45 & 12.3 \\
9 & 6.8 & 0.43 & 11.6 \\
10 & 7.9 & 0.44 & 12.1 \\
11 & 4.6 & 0.41 & 10.8 \\
12 & 2.8 & 0.40 & 10.5 \\
13 & 3.8 & 0.14 & 2.3 \\
elute & 1.0 & - & - \\
\hline
\end{tabular}

a Sample, No. 9; Flow rate, $23 \mathrm{ml} / \mathrm{hr}$; Total amount of the sample, $655.8 \mathrm{mg}$; Yield, $107 \%$ (A small amount of glue from a pressure sensitive tape may have been dissolved in the sample solution when the column was cut into 13 pieces.). The fraction number is given from the higher temperature side to the lower one. be expected to remain deposited on the glass beads in the column. Mixture No. 9 was developed by this method and the column was cut into 13 parts. The amount and molecular weight of the polymers deposited on the glass beads in each section were determined. The results obtained are shown in Table III. It can be seen that there is a discontinuous boundary at section No. 5, above which all polymers deposited have molecular weights $3 \times 10^{4}$ and below which $12 \times 10^{4}$. Moreover, despite the fact that about $32.5 \%$ of the initial amount of the sample remained on the glass beads coated initially (section No. 1), a clear single peak is shown in the sedimentation pattern. From these results, it may be concluded that reprecipitation really occurred due to the thermal gradient but that the efficiency of fractionation at the first stage was so high that almost complete separation of the components of the mixture was carried out at the first stage.

\section{Gel Permeation Chromatography}

In Figure 2, the apparent molecular weight distribution of a mixture (No. 9) calculated from GPC is compared with those of its components. Although a slight variation is observed between the peaks of the higher molecular weight component, the agreement is quite satisfactory. However the resolution of GPC is not sufficiently high to enable the two peaks in the chromatogram of sample No. 11 to be distinguished.

\section{Sedimentation Velocity Method}

This method appears to have the highest resolution of all methods employed here, since this method was able to show the double peaks in the sedimentation pattern of sample No. 11 which could not be shown by any other method. The integral distribution function of apparent sedimentation coefficient $G^{*}\left(S_{c}{ }^{*}, t, C_{0}\right)$ is obtained from $^{17}$

$$
G^{*}\left(S_{c}^{*}, t, C_{0}\right)=\int_{r_{0}}^{r} r^{2}\left(\frac{\partial n}{\partial r}\right) d r / \int_{r_{0}}^{\infty} r^{2}\left(\frac{\partial n}{\partial r}\right) d r
$$

as a function of time $t$. Here, $n$ is refractive index of the solution at a position $r$ and time $t$, and $r_{0}$ is the radial distance from the center of rotation to the air-liquid meniscus. From the curves of $G^{*} v s . r$ thus obtained for dif- 


\section{Determination of Molecular Weight Distribution}

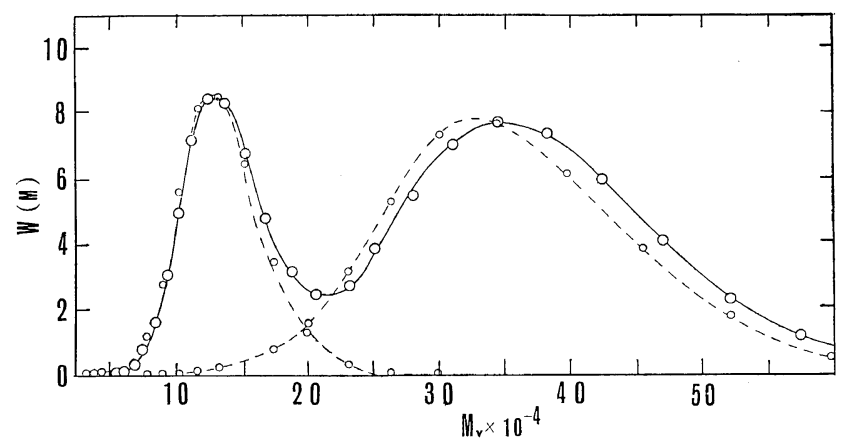

Figure 2. Molecular weight distribution of mixture No. 9 determined by gel permeation chromatography.

Unbroken line denotes the molecular weight distribution of sample No. 9, while broken lines denote the molecular weight distributions of the components, sample No. 2 and sample No. 4.

ferent $t$, we find $S_{c}{ }^{*}$ for a specified $G^{*}$ as a function of $t$, using the following equation:

$$
S_{c}{ }^{*}=\frac{\ln \left(\frac{r}{r_{0}}\right)}{\omega^{2}\left(t-t_{0}\right)}
$$

where $\omega$ is the angular velocity of the rotor, $t$ is the time observed and $t_{0}$ is the zero time correction. The zero time correction $t_{0}$ can be determined by extrapolating $\left(r_{h}{ }^{2}-r_{0}{ }^{2}\right) v s . t$ plot to $r_{h}=r_{0}, r_{h}$ being the position of peak. ${ }^{18,8}$

The values of $S_{c}^{*}$ at a specified value of $G^{*}$ thus obtained is plotted against $1 / t$ and extrapolated to $1 / t=0$ to eliminate the diffusion effect. An example for sample No. 11 is shown in Figure 3. In order to eliminate the effect of the concentration dependence of the sedimentation coefficient and the Johnston-Ogston effect, moreover, we should extrapolate the graph of $G\left(S_{c}\right.$, $\left.1 / t=0, C_{0}\right) v s . S_{c}$ for various $C_{0}$ to infinite dilution. In this work, however, $G\left(S_{0}, 1 / t=0\right.$, $\left.C_{0}=0\right)$ at infinite dilution was estimated from a graph of $\mathrm{G}$ obtained at a single concentration by using a modified method of Kotaka and Donkai. ${ }^{12}$ That is, the concentration dependence of the sedimentation coefficient is given by

$$
S_{c}=\frac{S_{0}}{1+k_{s} C_{0}}
$$

If we know $k_{s}$, we can estimate $S_{0}$ from an experiment at a single concentration. In general,

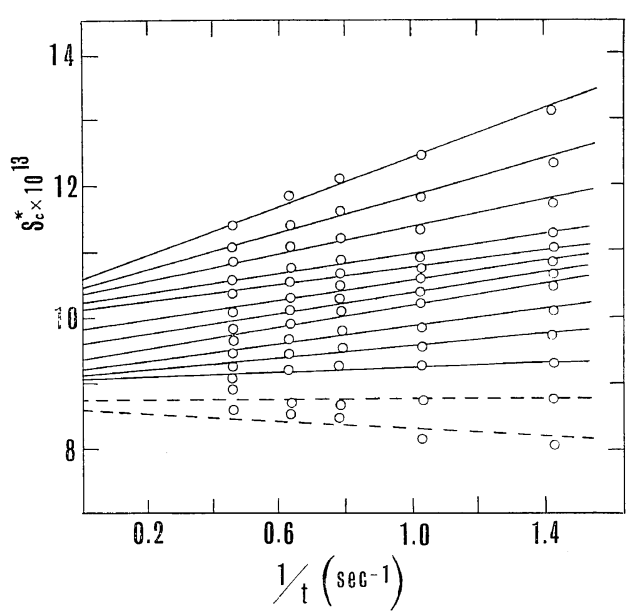

Figure 3. Plots of $S_{c}^{*} v s$. $1 / t$ for various values of $G^{*}$. Sample, No. 11. Initial concentration $C_{0}$, $0.254 \mathrm{~g} / \mathrm{d} l$. Rotor speed, $59780 \mathrm{rpm}$. The values of $G^{*}$ are $0.975,0.900,0.800,0.700,0.650,0.600$, $0.550,0.500,0.400,0.300,0.100$, and 0.025 from top to bottom.

Eq. 4 holds for sedimentation velocity of the center of mass, but in multi-component systems where the sedimentation pattern has double peaks, $k_{s}$ for each component is affected by the mixing ratio of the components. ${ }^{6}$ In $\theta$-solvents, however, it has been experimentally shown that $k_{s}$ for the center of mass may be used in practice for the peak of a constituent component in a mixture if the concentration at the position of 


\section{A. Yаmamoto, I. Noda, and M. Nagasawa}

the component is inserted into $C_{0}$ in Eq. 4, i.e., if the total concentration of the mixture is used for the faster component, while the concentration of the component is used for the slower component. ${ }^{6}$

The limiting sedimentation coefficient $S_{0}$ thus However, it should be noted that this is only

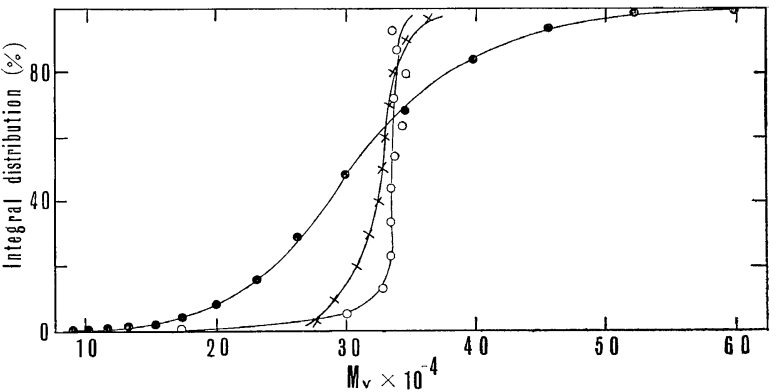

Figure 4. Molecular weight distributions of monodisperse polymer No. 4 determined by various methods.

White and black circles and crosses denote the molecular weight distributions by precipitation chromatography, GPC and sedimentation velocity methods, respectively.

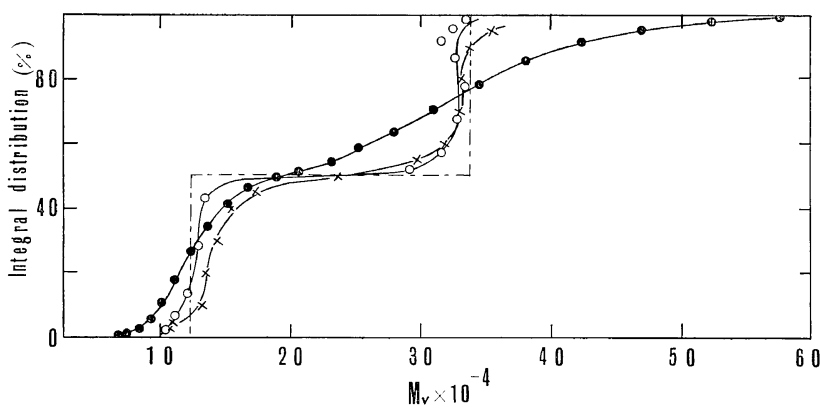

Figure 5. Molecular weight distributions of mixture No. 9 determined by various methods. Notations are the same as in Figure 4.

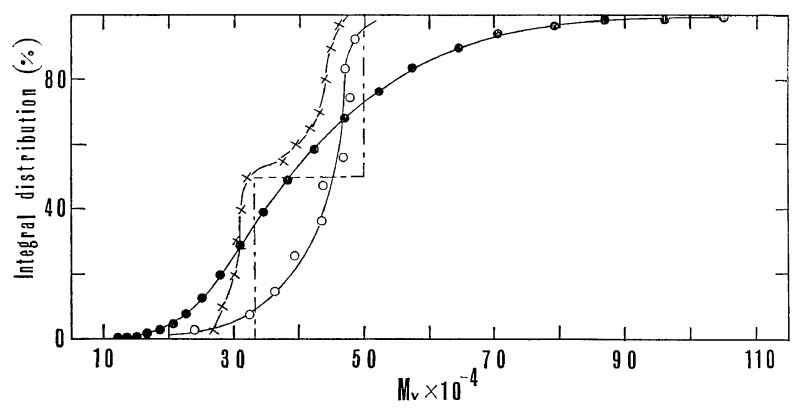

Figure 6. Molecular weight distributions of mixture No. 11 determined by various methods. Notations are the same as in Figure 4. 
an approximate equation determined from the sedimentation coefficients of the monodisperse samples in this work without correction for pressure effect. The numerical factor in Eq. 5 is different from that in Eq. 11 in ref. 7 which was determined after making a correction for the pressure effect. The molecular weight distributions of samples Nos. 4, 9 and 11 thus determined by the sedimentation velocity method are shown in Figures 4, 5 and 6, respectively.

\section{Comparison of Molecular Weight Distributions} Determined by Various Methods

The molecular weight distributions determined by the precipitation chromatography, gel permeation chromatography and the sedimentation velocity methods are summarized in Figures 46, for comparison with each other and also to be compared with the true molecular weight distributions calculated from those of the constituent components in the mixtures. In Figure 4 , an example for a monodisperse polymer No. 4 is shown, while in Figures 5 and 6, the experimental results for blended samples Nos. 9 and 11 are shown. From these figures we may conclude that both precipitation chromatography and the sedimentation method show the double peaks much better than the gel permeation chromatography method. Moreover, the sedimentation velocity method appears to have slightly higher resolution than the column fractionation method. Gel permeation chromatography apparently gives too broad molecular weight distributions for monodisperse polymers if no correction for the broadening effect is made. The present experimental results, however, do not necessarily mean that GPC has a resolution lower than the other methods when the samples are polydisperse.

Acknowledgement. We wish to thank Professor A. Kotera and Mr. Okamoto for allow- ing us to use a Waters Assoc. Model 200 and, moreover, for their competent advice concerning gel permeation chromatography. Our thanks are also due to Mr. T. Fujimoto who prepared experimental samples and to Mr. K. Kondo who carried out the experiments using the elution method.

\section{REFERENCES}

1. J. C. Moore, J. Polym. Sci., Part A, 2, 835 (1964).

2. L. H. Tung, J. Appl. Polym. Sci., 10, 375 (1966).

3. L. H. Tung, J. C. Moore, and G. W. Knight, J Appl. Polym. Sci., 10, 1261 (1966).

4. L. H. Tung, ibid., 10, 1271 (1966).

5. T. Fujimoto, N. Ozaki, and M. Nagasawa, $J$. Polym. Sci., Part A, 3, 2259 (1965).

6. A. Soda, T. Fujimoto, and M. Nagasawa, $J$. Phys. Chem., 71, 4274 (1967).

7. I. Noda, Y. Yamada, and M. Nagasawa, ibid., 72, 2890 (1968).

8. I. Noda, S. Saito, T. Fujimoto, and M. Nagasawa, ibid., 71, 4048 (1967).

9. C. A. Baker, and R. J. P. Williams, J. Chem. Soc., 2352 (1956).

10. N. S. Schneider, L. G. Holmes, C. F. Mijal, and J. D. Loconti, J. Polym. Sci., 37, 551 (1959).

11. G. V. Schulz, A. Scholz, and R. V. Figini, Makromol. Chem., 57, 220 (1962).

12. N.S. Schneider, J. D. Loconti, and L. G. Holmes, J. Appl. Polym. Sci., 5, 354 (1961).

13. G. V. Schulz, Z. Phys. Chem., B47, 155 (1940).

14. T. Kato, K. Miyaso, and M. Nagasawa, $J$. Phys. Chem., 72, 2161 (1968).

15. T. Kotaka, and N. Donkai, J. Polym. Sci., Part A-2, 6, 1457 (1968).

16. N. S. Schneider, J. Polym. Sci., Part C, 8, 179 (1965).

17. T. Homma, K. Kawahara, H. Fujita, and M. Ueda, Makromol. Chem. 67, 132 (1963).

18. H. G. Elias, Markomol. Chem., 24, 30 (1959). 